\title{
An Evolutionary Algorithm based Pattern Search Approach for Constrained Optimization
}

\author{
Rituparna Datta \\ Department of Mechanical Engineering, \\ Indian Institute of Technology \\ Kanpur, PIN: 208018, INDIA \\ Email: rdatta@iitk.ac.in \\ Kalyanmoy Deb, IEEE Fellow \\ Department of Mechanical Engineering, \\ Indian Institute of Technology \\ Kanpur, PIN: 208018, INDIA \\ Email: deb@iitk.ac.in
}

\author{
M. Fernanda P. Costa \\ Centre of Mathematics, \\ University of Minho \\ 4800-058 Guimaraes, PORTUGAL \\ Email: mfc@math.uminho.pt
}

\author{
A. Gaspar-Cunha, IEEE Member \\ Institute of Polymer and Composites/I3N, \\ University of Minho \\ 4800-058 Guimaraes, PORTUGAL \\ Email: agc@dep.uminho.pt
}

\begin{abstract}
Constrained optimization is one of the popular research areas since constraints are usually present in most real world optimization problems. The purpose of this work is to develop a gradient free constrained global optimization methodology to solve this type of problems. In the methodology proposed, the single objective constrained optimization problem is solved using a Multi-Objective Evolutionary Algorithm (MOEA) by considering two objectives simultaneously, the original objective function and a measure of constraint violation. The MOEA incorporates a penalty function where the penalty parameter is estimated adaptively. The use of penalty function method will enable to further improve the current best solution by decreasing the level of constraint violation, which is made using a gradient free local search method. The performance of the proposed methodology was assessed on a set of benchmark test problems. The results obtained allowed to conclude that the present approach is competitive when compared with other methods available.
\end{abstract}

\section{InTRODUCTION AND Motivation}

$\mathbf{T}$ HERE are many optimization problems, manly in the field of economics, engineering, decision science and operations research, where the objective function and/or some constraint functions can be formulated as non-convex and nonlinear functions. Application examples include areas like transportation, signal processing, production planning, robotics, project management, structural optimization, and VLSI design etc. [1], [2], [3] to name a few. The main motivation of the present work is to develop an efficient methodology to obtain a global solution for these type of optimization problems.

The mathematical formulation of the problem is:

$$
\begin{array}{cl}
\text { minimize } & f(\boldsymbol{x}), \\
\text { subject to } & g(\boldsymbol{x}) \geq 0, \\
& x \in \Omega
\end{array}
$$

where $f: \mathbb{R}^{n} \rightarrow \mathbb{R}$ and $g: \mathbb{R}^{n} \rightarrow \mathbb{R}^{m}$ are nonlinear continuous functions defined on the search space $\Omega \subseteq \mathbb{R}^{n}$. Usually, the search space $\Omega$ is defined as $\Omega=\left\{x \in \mathbb{R}^{n}:-\infty<l \leq x \leq\right.$ $u<\infty$. Problems with equality constraints, $h(x)=0$, are reformulated into the above form using a couple of inequality constraints $h(x)+\gamma \geq 0$ and $-h(x)+\gamma \geq 0$, where $\gamma$ represents a positive small tolerance $(0<\gamma \ll 1)$. The set $\mathbf{F}=\{x \in \Omega$ : $g(x) \geq 0\}$ defines the feasible region. Since, it is not assumed that the objective and constraint functions are convex, many global and local solutions can exist in the set $\mathbf{F}$.

Initially, evolutionary algorithms (EAs) were designed to solve global unconstrained optimization problems, after being extended to handle constraints [4], [5], [6], [7]. One of the most popular and simple class of methods to solve globally non-convex constrained optimization problems are based on penalty functions [4], [8]. In these methods, the penalty function is defined combining a measure of constraint violation with the objective function.

A penalty function method works by increasing the fitness value of the infeasible solutions proportionally to their level of constraint violation. Some of the penalty function based evolutionary research works are available in [9], [10], [11]. One of the drawbacks of the penalty function method is that, it needs a proper estimation of penalty parameter to handle the constraints efficiently, throughout the iterative process. If the penalty parameter is too large, an arbitrary feasible solution can be returned. On the other hand, if the parameter is too small, more emphasis is given to the objective function and, thus, the constraints can be neglected, which can result in an infeasible solution. These drawback of the penalty function approach motivated researchers to develop alternative methods to deal with constraints in global optimization problems.

Deb in [7] proposed a penalty-parameter-less EA approach which efficiently handles constraints using the following criteria: (i) if there are two feasible solutions, the one with less objective function value is selected, (ii) if there are two solutions, of which one feasible and the other infeasible, the feasible solution is selected, (iii) if there are two infeasible solutions, the one with less constraint violation is selected. Some other penalty parameter less constraint handling approaches 
are available in [5], [12], [13], [14], [15].

In addition to the penalty function approach, another idea, that received the attention among evolutionary research community, was to convert the constrained optimization problem into a bi-objective optimization problem. In the bi-objective approach two objectives are simultaneously minimized, one is a measure of the constraint violation and the other is the original objective function. Coello in [16] proposed an approach in which all constraints are treated as objectives. Herein, instead to solve a bi-objective problem, the method solved a multi-objective problem. However, this idea is not always appropriate in real world scenarios, since the complexity of the problem increases considerably with the number of constraints. Some other studies in bi-objective based constraint handling approaches can be found in [6], [17], [18], [19], [20], [21].

Although evolutionary based optimization methods have proven their efficiency in a large number of problems, they have the weakness of exact convergence. To overcome this issue some hybrid evolutionary algorithms have been proposed. Usually, EAs are coupled with other optimization techniques or heuristic methods. To perform this hybridization both the techniques are integrated intelligently to retain the good properties of both techniques. Some hybrid evolutionary methods are available in [22], [23], [24], [25], [26].

Recently in [27], to solve non-convex and non-linear constrained global optimization using an evolutionary technique, the constrained optimization problem was converted into a biobjective problem:

$$
\min _{x \in \Omega}(f(x), \theta(x)),
$$

where $\theta$ is a non-negative continuous aggregate constraint violation function defined by

$$
\theta(x)=\sum_{j=0}^{m}\left|\min \left\{g_{j}(x), 0\right\}\right| .
$$

In this approach, a penalty function method is applied to improve the performance EA. Herein, at pre-defined generations of EA, a penalty function is solved by a local approach. First, a cubic polynomial is fitted (using a nonlinear least square formulation) to a set of non-dominated solutions, that were obtained between the measure of the constraint violation and the objective function - the Pareto-optimal front. The slope of this polynomial is used as an approximation to the penalty parameter. Thereafter, given as initial point the best current point (the lesser infeasible point in the Pareto front), the penalty function is solved by a local gradient based approach. Finally, the minimizer of the penalty function is used to replace the worst point in the current Pareto front. This process is repeated until convergence is achieved.

The structure of the present paper is as follows; in section II the details of the proposed hybrid evolutionary coupled with a pattern search method is described, hereafter called EA-PS method. In section III, we report the results of the numerical experiments with a set of benchmark problems. Finally, the paper finishes with conclusions and future work in section IV.

\section{Proposed Hybrid Evolutionary and Pattern Search} METHOD

In this section the hybrid methodology (EA-PS) used to compute the global solution of problem (1) is described. The hybridization is made by coupling an evolutionary algorithm with a gradient free pattern search method to optimize the penalized function.

\section{A. No Gradient Information}

In [27] the local search uses gradient information to optimize the penalty function. However, often the gradient information may not be available. For instance, in black-box applications the gradient information of constraints and the objective function are not available and are forbidden to be used. In such situations the herein proposed derivative free local search integrated into the EA, target these type of optimization problems. Therefore in this work, constrained optimization problems are solved using a derivative free method.

\section{B. Pattern Search for Bound Constrained Problems}

Direct search methods for unconstrained optimization problems generate a sequence of points $\left\{x_{k}\right\}$ in $\mathbb{R}^{n}$ with nonincreasing objective function values. At each iteration, the objective function is computed at a finite set of trial points to try to find one that yields a lower objective function than the current point. Direct search methods works without using any gradient information and additionally not any derivative approximation is made. Pattern search are one of the popular direct methods in which trial points are computed follow an exact calculations. In the present work we apply a pattern search method, more specifically the Hooke and Jeeves pattern search method [28], to minimize the penalty function:

$$
P(\boldsymbol{x})=f(\boldsymbol{x})+r \theta(\boldsymbol{x}),
$$

where $r \geq 0$ is the penalty parameter.

In this section we describe details related to our implementation of this method, in particular, the scheme used to keep the iterates in the set $\Omega$ and the termination criteria. In the Hooke-Jeeves method two types of movements are performed iteratively, namely exploratory moves and heuristic pattern moves. In the exploratory move a coordinate search with a step length of $\Delta_{k}$ around the current point $x_{k}$ is performed. Herein, one coordinated at time of the current point $x_{k}$ is modified along of positive and negative coordinate directions and the best point (a point with a lower function value) is recorded. The point is updated to the best position at each variable modification. The iteration is considered successful if a best point $\hat{x}_{k+1}$ is found at the end of all variables modifications. Otherwise it is an unsuccessful iteration and the step length $\Delta_{k}$ is reduced.

When the iteration is successful the current and the best points are used to make a pattern move. The $\hat{x}_{k+1}-x_{k}$ entity defines a promising direction and the pattern search move jump from the best point $\hat{x}_{k+1}$ along that direction and it carries out an exploratory move around the new trial point 
$\hat{x}_{k+1}+\left(\hat{x}_{k+1}-x_{k}\right)$ instead of the current best $\hat{x}_{k+1}$. Thereafter, in case of a successful exploratory move, a new best point is accepted. Otherwise, in case of an unsuccessful exploratory move, the pattern search move is not accepted, and the method reduces to an exploratory move around $x_{k+1} \leftarrow \hat{x}_{k+1}$.

In order to maintain the iterates in set $\Omega$ in the Hooke Jeeves pattern search method, the iterates are projected into this set component-wise, $\left(x_{k}\right)_{i}=\max \left(l_{i}, \min \left(\left(x_{k}\right)_{i}, u_{i}\right)\right)$ for $i=1, \cdots, n$. To deal with variables with different magnitude, the Hooke Jeeves algorithm implementation uses a step length vector $\Delta$. Given an initial guess $x_{0} \in \Omega$, the vector $\Delta_{0}$ is initialized component-wise as follows:

$$
\left(\Delta_{0}\right)_{i}= \begin{cases}\rho\left(x_{0}\right)_{i}, & \text { if }\left(x_{0}\right)_{i} \neq 0 \\ \rho, & \text { otherwise }\end{cases}
$$

where $\rho$ is a positive parameter. Let $\alpha>1$ be a step reduction factor. The stopping criterion of the pattern search method is defined by $\left\|\Delta_{k}\right\|<\epsilon$, where $\epsilon>0$ is the termination parameter. The Hooke-Jeeves pattern search method is described in Algorithm 1.

\section{Hybrid EA-PS method}

Flowchart 1 describes the steps of the proposed approach. First, a single objective constrained optimization is converted into a bi-objective problem. Here, Non-dominated Sorting Genetic Algorithm-II (NSGA-II) [29] is used to solve the bi-objective problem and for obtaining the Pareto-optimal front. After every 5 generations, non-dominated solutions are identified and a cubic polynomial is fitted to those nondominated solutions. The slope of this cubic polynomial is used to estimate the penalty parameter of (2). Taking the best current point as the initial guess, the penalty function (2) is minimized using the Hooke and Jeeves pattern search method. The optimal solution of the penalty function is used to replace the worst point in the current Pareto-optimal front. This process is repeated until two consecutive optimal local searched solutions of the penalty functions are less than small positive tolerance and the hybrid EA-PS stops.

\section{Simulation Results and Discussions}

To validate the proposed EA-PS, a set of six problems is used, out of which five are shown in the Appendix A. One of these problems is shown below.

The $\mathrm{C}$ programming is used for the evolutionary algorithm and Hooke and Jeeves is implemented in Matlab. The simulations are performed on a PC with $2.1 \mathrm{GHz}$ Intel core i3 and 2 GB of RAM. The parameters have been set as follows after an empirical study:

Population size $=16 n$,

SBX probability $=0.9$,

SBX index $=10$,

Polynomial mutation probability $=1 / n$, and

Mutation index $=100$.

The hybrid algorithm is allowed to runs 50 times with different initial populations. First, EA-PS is tested in a two

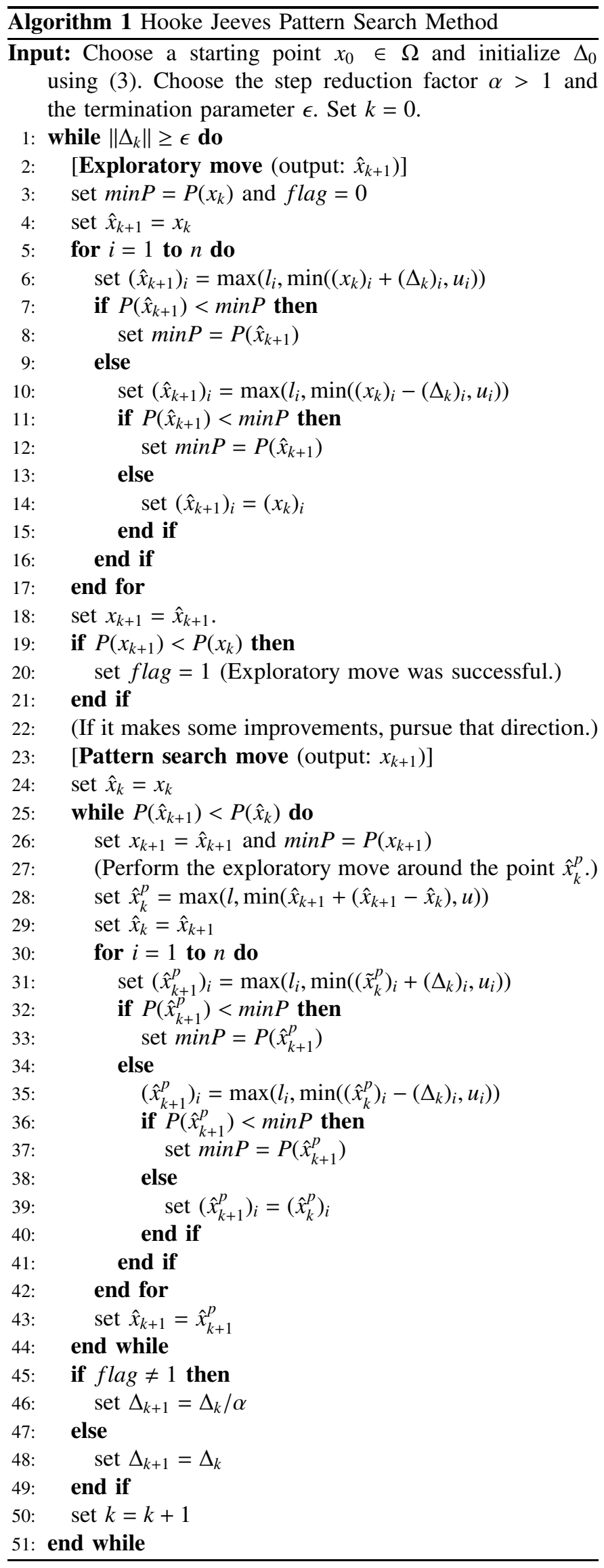




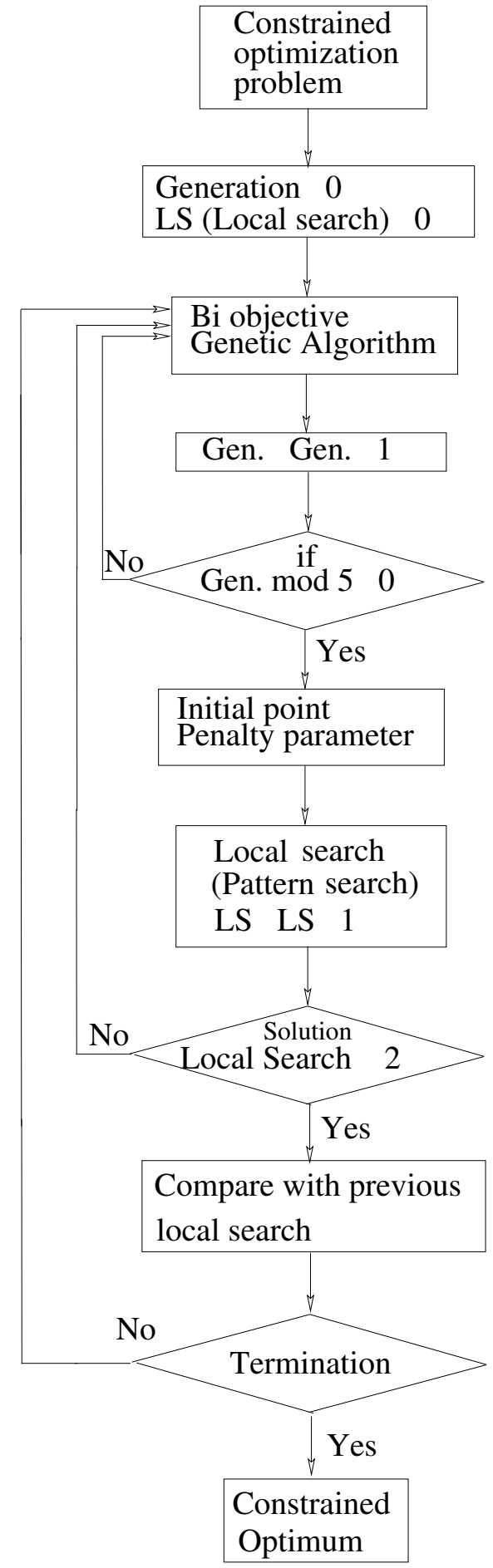

Fig. 1. Flowchart of the proposed EA-PS method.

variable problem. Thereafter, the efficiency of the algorithm is tested with the remaining five problems. When difference between the absolute values of two consecutive local searched solutions are less than $10^{-4}$ we terminated the algorithm.

\section{A. Problem PI}

First, the following two-variable problem is tested. The problem has two inequality constraints. The constraints are non-linear and non-convex and the first one is active at the optimum [27]:

$$
\begin{array}{cl}
\operatorname{minimize} & f(\mathbf{x})=\left(x_{1}-3\right)^{2}+\left(x_{2}-2\right)^{2} \\
\text { subject to } & g_{1}(\mathbf{x}) \equiv 4.84-\left(x_{1}-0.05\right)^{2}-\left(x_{2}-2.5\right)^{2} \geq 0 \\
& g_{2}(\mathbf{x}) \equiv x_{1}^{2}+\left(x_{2}-2.5\right)^{2}-4.84 \geq 0 \\
& 0 \leq x_{1} \leq 6 \\
& 0 \leq x_{2} \leq 6
\end{array}
$$

Table I shows the total number of function evaluations (FE), which is the sum of the number of function evaluations taken by EA and the Hooke-Jeeves method, and the corresponding objective function values (f). We compare the results with the previous hybrid method [27] that uses gradient information. The Table I clearly shows that our best number of function evaluation is better than the previous reported one. However, in terms of median and worst of the number of function evaluations the previous method outperform the EA-PS, which is expected since EA-PS does not use gradient information. But the results are comparable. We can conclude that EA-PS method performs successfully.

TABLE I

Function EVALUATIONS, FE (NSGA-II AND LOCAL SEARCH) AND OPTIMAL SOLUTION, BY THE EARLIER APPROACH AND EA-PS IN 50 RUNS.

\begin{tabular}{|l|c|c|c|c|}
\hline & & Best & Median & Worst \\
\hline Single & FE & $677(600+77)$ & $733(600+133)$ & $999(900+99)$ \\
Penalty [27] & $f$ & 0.627380 & 0.627379 & 0.627379 \\
\hline EA-PS & FE & $672(600+72)$ & $1,342(1,200+142)$ & $3,332(3,000+332)$ \\
& $f$ & 0.627485 & 0.627424 & 0.628774 \\
\hline
\end{tabular}

Table II shows similar results for other five problems. In Table II we compare our results with the results obtained by three previously developed evolutionary algorithms based constraint handling techniques. This comparison is again made in terms of total number of function evaluations and the corresponding objective values.

Table III reports similar results obtained by seven proposed approaches namely HM: Homomorphous Mapping, SR: Stochastic Ranking, ASCHEA: Adaptive Segregational Constraint Handling Evolutionary, SMES: Simple Multimembered Evolution Strategy, FSA: Filter Simulated Annealing, ATMES: Adaptive Trade-off Model Evolution Strategy, and NM-PSO: Nelder-Mead Particle Swarm Optimization [30], [31], [32], [33], [34], [35], [36]. Based on the results we may conclude that EA-PS has a good performance. EAPS is able to reach the global optimal solution with the desired accuracy, beside using any gradient information, except with problems TP4 and TP8. 
TABLE II

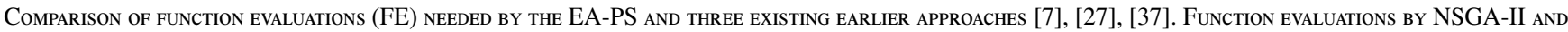
LOCAL SEARCH HAVE BEEN SHOWN SEPARATELY.

\begin{tabular}{|c|c|c|c|c|c|c|}
\hline \multirow[t]{2}{*}{ Problem } & \multicolumn{3}{|c|}{ Penalty Parameter Less Approach [7] } & \multicolumn{3}{|c|}{ Single Penalty Approach [27] } \\
\hline & Best & Median & Worst & Best & Median & Worst \\
\hline TP3 (FE) & 65,000 & 65,000 & 65,000 & 2,427 & 4,676 & 13,762 \\
\hline NSGA-II+Local & & & & $2,000+427$ & $3,000+1,676$ & $11,000+2,762$ \\
\hline$\left(f^{*}\right)$ & -15 & -15 & -13 & -15 & -15 & -12 \\
\hline $\mathrm{TP} 4(\mathrm{FE})$ & 320,080 & 320,080 & 320,080 & 31,367 & 54,946 & 100,420 \\
\hline NSGA-II+Local & & & & $14,400+16,967$ & $24,600+30,346$ & $45,600+54,820$ \\
\hline$\left(f^{*}\right)$ & $7,060.221$ & $7,220.026$ & $10,230.834$ & $7,078.625$ & $7,049.943$ & $7,940.678$ \\
\hline TP5 (FE) & 350,070 & 350,070 & 350,070 & 6,332 & 15,538 & 38,942 \\
\hline NSGA-II+Local & & & & $3,920+2,412$ & $9,520+6,018$ & $25,200+13,742$ \\
\hline$\left(f^{*}\right)$ & 680.634 & 680.642 & 680.651 & 680.630 & 680.634 & 680.876 \\
\hline TP6 (FE) & 250,000 & 250,000 & 250,000 & 1,120 & 2,016 & 6,880 \\
\hline NSGA-II+Local & & & & $800+320$ & $1,200+816$ & $3,600+3,280$ \\
\hline$\left(f^{*}\right)$ & $-30,665.537$ & $-30,665.535$ & $-29,846.654$ & $-30,665.539$ & $-30,665.539$ & $-30,649,552$ \\
\hline TP8 (FE) & 350,000 & 350,000 & 350,000 & 4,880 & 23,071 & 83,059 \\
\hline NSGA-II+Local & & & & $3,200+1,680$ & $8,000+5,071$ & $44,800+38,259$ \\
\hline$\left(f^{*}\right)$ & 24.372 & 24.409 & 25.075 & 24.308 & 25.651 & 31.254 \\
\hline \multirow[t]{2}{*}{ Problem } & \multicolumn{3}{|c|}{ Adaptive Normalization Approach [37] } & \multicolumn{3}{|c|}{ EA-PS } \\
\hline & Best & Median & Worst & Best & Median & Worst \\
\hline TP3 (FE) & 2,333 & 2,856 & 11,843 & 2,959 & 5,752 & 32,292 \\
\hline NSGA-II+Local & $2,000+333$ & $2,000+856$ & $8,000+3,843$ & $2,000+959$ & $3,000+1,702$ & $25,000+7,292$ \\
\hline$\left(f^{*}\right)$ & -12 & -15 & -15 & -14.968 & -14.993 & -14.992 \\
\hline TP4 (FE) & 2,705 & 27,235 & $1,07,886$ & 10,064 & 37,724 & $1,24,128$ \\
\hline NSGA-II+Local & $1,200+1,505$ & $7,200+20,035$ & $45,600+62286$ & $9,600+464$ & $36,000+1,724$ & $87,600+36,528$ \\
\hline$\left(f^{*}\right)$ & $7,049.588$ & $7,059.576$ & $7,065.348$ & $8,200.0697$ & 7078.2195 & 7117.6887 \\
\hline TP5 (FE) & 1,961 & 11,729 & 42,617 & 3,222 & 6,682 & 13,379 \\
\hline NSGA-II+Local & $1,120+841$ & $7,280+4,449$ & $27,440+15,177$ & $2,800+422$ & $5,040+1,582$ & $8,960+4,419$ \\
\hline$\left(f^{*}\right)$ & 680.635 & 680.631 & 680.646 & 680.6387 & 681.6397 & 681.0874 \\
\hline TP6 (FE) & 1,123 & 4,183 & 13,631 & 8,396 & 12,679 & 18,327 \\
\hline NSGA-II+Local & $800+323$ & $2,400+1,783$ & $8,400+5,231$ & $8,000+396$ & $12,000+679$ & $16,000+2,327$ \\
\hline$\left(f^{*}\right)$ & $-30,665.539$ & $-30,665.539$ & $-30,665.539$ & -30665.530 & -30665.540 & -30665.540 \\
\hline TP8 (FE) & 7,780 & 68,977 & $3,54,934$ & 8,712 & 85,324 & $\mathbf{1 , 8 5 , 2 7 3}$ \\
\hline NSGA-II+Local & $5,600+2,180$ & $41,600+27,377$ & $1,600+1673$ & $7,200+1,512$ & $64,000+21,324$ & $1,28,000+57,273$ \\
\hline$\left(f^{*}\right)$ & 24.565 & 24.306 & 24.306 & 25.889 & 27.309 & 31.146 \\
\hline
\end{tabular}




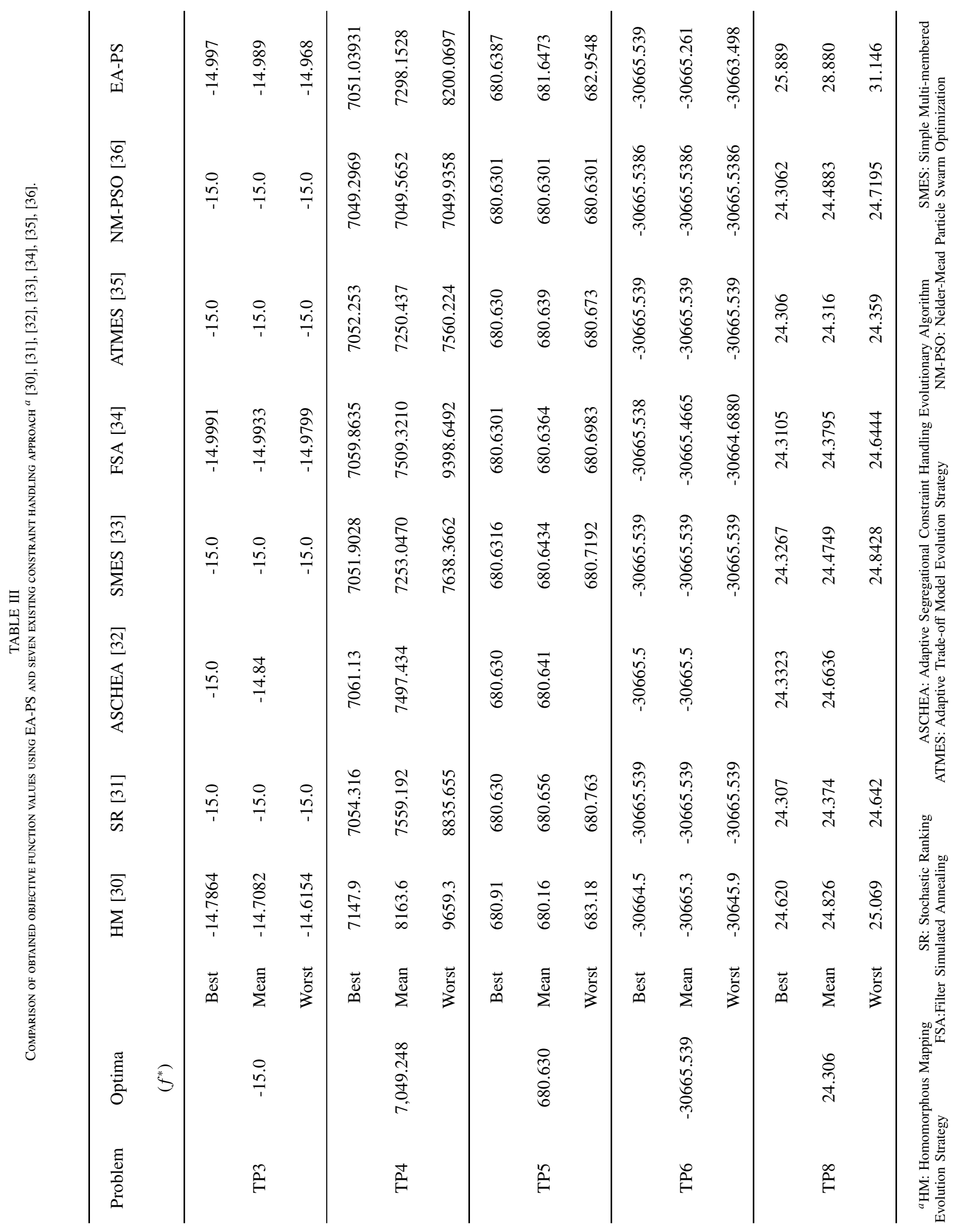




\section{Conclusions}

A hybrid evolutionary approach coupled with a pattern search method for global nonlinear constrained optimization is proposed. The advantage of the this method lies on the fact that the local search does not need any gradient information, which may not be available in many instances. In the proposed hybrid method, an evolutionary algorithm is used to generate the Pareto-optimal front. At every five generations, a penalty function is minimized, in which its penalty parameter is estimated by the slope of a cubic polynomial that is fitted to the points defined by the Pareto-front. To minimize the penalty function, Hooke and Jeeves pattern search method is used, taking as initial point the best current point in the Pareto-optimal set (the point which has the lesser constraint violation measure). The minimizer of the penalty function is used to replace the worst point in the Pareto-front. The proposed method is tested with a set of six constrained optimization problems very well known in literature. In the test, the robustness of the hybrid algorithm is tested using different initial populations. The total number of function evaluations is compared with three evolutionary based constraint handling methods. In addition to that the best, average and the worst objective function value is also compared with seven previously developed methods. Results show that the proposed hybrid method is efficient. Since most practical problems are expected to be non-differentiable or discrete, evolutionary algorithms are better off in hybridizing with gradient-free methods, such as Hooke-Jeeves method. The results here are promising and the combined method needs further testing and analysis. In future we plan to apply it to solve problems having equality constraints and some real life constrained optimization problems.

\section{APPENDIX}

\section{A. Problem TP3}

$$
\begin{aligned}
\min . & f(\mathbf{x})=5 \sum_{i=1}^{4} x_{i}-5 \sum_{i=1}^{4} x_{i}^{2}+5 \sum_{i=5}^{13} x_{i}, \\
\text { s.t. } & g_{1}(x) \equiv 2 x_{1}+2 x_{2}+x_{10}+x_{11}-10 \leq 0, \\
& g_{2}(x) \equiv 2 x_{1}+2 x_{3}+x_{10}+x_{12}-10 \leq 0, \\
& g_{3}(x) \equiv 2 x_{2}+2 x_{3}+x_{11}+x_{12}-10 \leq 0, \\
& g_{4}(x) \equiv-8 x_{1}+x_{10} \leq 0, \\
& g_{5}(x) \equiv-8 x_{2}+x_{11} \leq 0, \\
& g_{6}(x) \equiv-8 x_{3}+x_{12} \leq 0, \\
& g_{7}(x) \equiv-2 x_{4}-x_{5}+x_{10} \leq 0, \\
& g_{8}(x) \equiv-2 x_{6}-x_{7}+x_{11} \leq 0, \\
& g_{9}(x) \equiv-2 x_{8}-x_{9}+x_{12} \leq 0,
\end{aligned}
$$

where $0 \leq x_{i} \leq 1$ for $i=1, \ldots, 9,0 \leq x_{i} \leq 100$ for $i=10,11,12$, and $0 \leq x_{13} \leq 1$. The minimum point is $\mathbf{x}^{*}=(1,1,1,1,1,1,1,1,1,3,3,3,1)^{T}$, where six constraints $\left(g_{1}, g_{2}, g_{3}, g_{7}, g_{8}\right.$ and $\left.g_{9}\right)$ are active and $f\left(\mathbf{x}^{*}\right)=-15$.

\section{B. Problem TP4}

The problem is given as follows:

$$
\begin{aligned}
\min . & f(\mathbf{x})=x_{1}+x_{2}+x_{3}, \\
\text { s.t. } & g_{1}(\mathbf{x}) \equiv-1+0.0025\left(x_{4}+x_{6}\right) \leq 0, \\
& g_{2}(\mathbf{x}) \equiv-1+0.0025\left(x_{5}+x_{7}-x_{4}\right) \leq 0, \\
& g_{3}(\mathbf{x}) \equiv-1+0.01\left(x_{8}-x_{5}\right) \leq 0, \\
& g_{4}(\mathbf{x}) \equiv-x_{1} x_{6}+833.33252 x_{4}+100 x_{1}-83333.333 \leq 0, \\
& g_{5}(\mathbf{x}) \equiv-x_{2} x_{7}+1250 x_{5}+x_{2} x_{4}-1250 x_{4} \leq 0, \\
& g_{6}(\mathbf{x}) \equiv-x_{3} x_{8}+1250000+x_{3} x_{5}-2500 x_{5} \leq 0, \\
& 100 \leq x_{1} \leq 10000,1000 \leq\left(x_{2}, x_{3}\right) \leq 10000, \\
& 10 \leq\left(x_{4}, \ldots, x_{8}\right) \leq 1000 .
\end{aligned}
$$

The minimum point lies at $\mathbf{x}^{*}=(579.307,1359.971$, $5109.971,182.018,295.601,217.982,286.417,395.601)^{T}$ with a function value $f^{*}=7049.280$. All constraints are active at this point.

\section{Problem TP5}

The problem is given as follows:

$$
\begin{array}{cl}
\min . & f(\mathbf{x})=\left(x_{1}-10\right)^{2}+5\left(x_{2}-12\right)^{2}+x_{3}^{4}+3\left(x_{4}-11\right)^{2} \\
& +10 x_{5}^{6}+7 x_{6}^{2}+x_{7}^{4}-4 x_{6} x_{7}-10 x_{6}-8 x_{7} \\
\text { s.t. } & g_{1}(\mathbf{x}) \equiv-127+2 x_{1}^{2}+3 x_{2}^{4}+x_{3}+4 x_{4}^{2}+5 x_{5} \leq 0 \\
& g_{2}(\mathbf{x}) \equiv-282+7 x_{1}+3 x_{2}+10 x_{2}^{3}+x_{4}-x_{5} \leq 0 \\
& g_{3}(\mathbf{x}) \equiv-196+23 x_{1}+x_{2}^{2}+6 x_{6}^{2}-8 x_{7} \leq 0 \\
& g_{4}(\mathbf{x}) \equiv 4 x_{1}^{2}+x_{2}^{2}-3 x_{1} x_{2}+2 x_{3}^{2}+5 x_{6}-11 x_{7} \leq 0 \\
& -10 \leq x_{i} \leq 10, \quad i=1, \ldots, 7
\end{array}
$$

The minimum is at $\mathbf{x}^{*}=(2.330,1.951,-0.478,-4.366$, $-0.624,1.038,1.594)^{T}$ with $f=680.630$. Constraints $g_{1}$ and $g_{4}$ are active at the minimum point.

\section{Problem TP6}

The problem is given as follows:

$$
\begin{array}{ll}
\min . \quad & f(\mathbf{x})=5.3578547 x_{3}^{2}+0.8356891 x_{1} x_{5}+37.293239 x_{1} \\
& -40792.141 \\
\text { s.t. } & g_{1}(\mathbf{x}) \equiv 85.334407+0.0056858 x_{2} x_{5}+0.0006262 x_{1} x_{4} \\
& \quad-0.0022053 x_{3} x_{5}-92 \leq 0 \\
& g_{2}(\mathbf{x}) \equiv-85.334407-0.0056858 x_{2} x_{5}-0.0006262 x_{1} x_{4} \\
& +0.0022053 x_{3} x_{5} \leq 0 \\
& g_{3}(\mathbf{x}) \equiv 80.51249+0.0071317 x_{2} x_{5}+0.0029955 x_{1} x_{2} \\
& +0.0021813 x_{3}^{2}-110 \leq 0 \\
& g_{4}(\mathbf{x}) \equiv-80.51249-0.0071317 x_{2} x_{5}-0.0029955 x_{1} x_{2} \\
& -0.0021813 x_{3}^{2}+90 \leq 0 \\
& g_{5}(\mathbf{x}) \equiv 9.300961+0.0047026 x_{3} x_{5}+0.0012547 x_{1} x_{3} \\
& +0.0019085 x_{3} x_{4}-25 \leq 0 \\
& g_{6}(\mathbf{x}) \equiv-9.300961-0.0047026 x_{3} x_{5}-0.0012547 x_{1} x_{3} \\
& -0.0019085 x_{3} x_{4}+20 \leq 0 \\
& 78 \leq x_{1} \leq 102,33 \leq x_{2} \leq 45,27 \leq\left(x_{3}, x_{4}, x_{5}\right) \leq 45 .
\end{array}
$$

The minimum is at $\mathbf{x}^{*}=(78,33,29.995,45,36.776)^{T}$ with a function value $f^{*}=-30665.539$. Constraints $g_{1}$ and $g_{6}$ are active at the minimum point. 


\section{E. Problem TP8}

The problem is given as follows:

$$
\begin{aligned}
& \text { min. } f(\mathbf{x})=x_{1}^{2}+x_{2}^{2}+x_{1} x_{2}-14 x_{1}-16 x_{2}+\left(x_{3}-10\right)^{2} \\
& \quad+4\left(x_{4}-5\right)^{2}+\left(x_{5}-3\right)^{2}+2\left(x_{6}-1\right)^{2}+5 x_{7}^{2}+7\left(x_{8}-11\right)^{2} \\
& \quad+2\left(x_{9}-10\right)^{2}+\left(x_{10}-7\right)^{2}+45, \\
& \text { s.t. } \\
& g_{1}(\mathbf{x}) \equiv-105+4 x_{1}+5 x_{2}-3 x_{7}+9 x_{8} \leq 0, \\
& g_{2}(\mathbf{x}) \equiv 10 x_{1}-8 x_{2}-17 x_{7}+2 x_{8} \leq 0, \\
& g_{3}(\mathbf{x}) \equiv-8 x_{1}+2 x_{2}+5 x_{9}-2 x_{10}-12 \leq 0 \\
& g_{4}(\mathbf{x}) \equiv 3\left(x_{1}-2\right)^{2}+4\left(x_{2}-3\right)^{2}+2 x_{2}^{3}-7 x_{4}-120 \leq 0, \\
& g_{5}(\mathbf{x}) \equiv 5 x_{1}^{2}+8 x_{2}+\left(x_{3}-6\right)^{2}-2 x_{4}-40 \leq 0 \\
& g_{6}(\mathbf{x}) \equiv x_{1}^{2}+2\left(x_{2}-2\right)^{2}-2 x_{1} x_{2}+14 x_{5}-6 x_{6} \leq 0, \\
& g_{7}(\mathbf{x}) \equiv 0.5\left(x_{1}-8\right)^{2}+2\left(x_{2}-4\right)^{2}+3 x_{5}^{2}-x_{6}-30 \leq 0, \\
& g_{8}(\mathbf{x}) \equiv-3 x_{1}+6 x_{2}+12\left(x_{9}-8\right)^{2}-7 x_{10} \leq 0, \\
& -10 \leq x_{i} \leq 10, \quad i=1, \ldots, 10 .
\end{aligned}
$$

The minimum is at $\mathbf{x}^{*}=(2.172,2.364,8.774,5.096,0.991$, $1.431,1.322,9.829,8.280,8.376)^{T}$ and function value 24.306 .

\section{REFERENCES}

[1] O. L. Frost III, "An algorithm for linearly constrained adaptive array processing," Proceedings of the IEEE, vol. 60, no. 8, pp. 926-935, 1972.

[2] R. Datta and K. Deb, "Multi-objective design and analysis of robot gripper configurations using an evolutionary-classical approach," in Proceedings of the 13th annual conference on Genetic and evolutionary computation. ACM, 2011, pp. 1843-1850.

[3] D. Merkle, M. Middendorf, and H. Schmeck, "Ant colony optimization for resource-constrained project scheduling," Evolutionary Computation, IEEE Transactions on, vol. 6, no. 4, pp. 333-346, 2002.

[4] R. Fletcher, "An ideal penalty function for constrained optimization," IMA Journal of Applied Mathematics, vol. 15, no. 3, pp. 319-342, 1975.

[5] D. Coit, A. Smith, and D. Tate, "Adaptive penalty methods for genetic optimization of constrained combinatorial problems," INFORMS Journal on Computing, vol. 8, pp. 173-182, 1996.

[6] S.-C. Hong and Y.-B. Park, "A heuristic for bi-objective vehicle routing with time window constraints," International Journal of Production Economics, vol. 62, no. 3, pp. 249-258, 1999.

[7] K. Deb, "An efficient constraint handling method for genetic algorithms," Computer Methods in Applied Mechanics and Engineering, vol. 186, no. 2-4, pp. 311-338, 2000.

[8] R. Haftka and J. Starnes, "Applications of a quadratic extended interior penalty function for structural optimization," in AIAA, ASME, and SAE, Structures, Structural Dynamics, and Materials Conference, 16 th, Denver, Colo, 1975, p. 1975.

[9] A. Homaifar, S. H.-V. Lai, and X. Qi, "Constrained optimization via genetic algorithms," Simulation, vol. 62, no. 4, pp. 242-254, 1994.

[10] Z. Michalewicz and C. Z. Janikow, "Handling constraints in genetic algorithms," in Proceedings of the Fourth International Conference on Genetic Algorithms, 1991, pp. 151-157.

[11] O. Yeniay, "Penalty function methods for constrained optimization with genetic algorithms," Mathematical and Computational Applications, vol. 10, no. 1, pp. 45-56, 2005.

[12] C. Coello, "Use of a self-adaptive penalty approach for engineering optimization problems," Computers in Industry, vol. 41, no. 2, pp. 113127,2000

[13] R. Farmani and J. A. Wright, "Self-adaptive fitness formulation for constrained optimization," Evolutionary Computation, IEEE Transactions on, vol. 7, no. 5, pp. 445-455, 2003.

[14] Z. Wu and T. Walski, "Self-adaptive penalty approach compared with other constraint-handling techniques for pipeline optimization," Journal of water resources planning and management, vol. 131, no. 3, pp. 181192,2005

[15] A. Eiben and Z. Ruttkay, "Self-adaptivity for constraint satisfaction: Learning penalty functions," in Evolutionary Computation, 1996., Proceedings of IEEE International Conference. IEEE, 1996, pp. 258-261.

[16] A. Carlos and C. Coello, "Treating constraints as objectives for singleobjective evolutionary optimization," Engineering Optimization+ A35, vol. 32, no. 3, pp. 275-308, 2000.
[17] P. D. Surry, N. J. Radcliffe, and I. D. Boyd, "A multi-objective approach to constrained optimisation of gas supply networks : The COMOGA method," in Evolutionary Computing. AISB Workshop. Springer-Verlag, 1995 , pp. $166-180$

[18] K. Deb, S. Lele, and R. Datta, "A hybrid evolutionary multi-objective and SQP based procedure for constrained optimization," in Proceedings of the 2nd International Conference on Advances in Computation and Intelligence (ISICA 2007). Springer-Verlag, 2007, pp. 36-45.

[19] R. Datta, "Constrained engineering design optimization using a hybrid bi-objective evolutionary-classical methodology," Simulated Evolution and Learning, pp. 633-637, 2010.

[20] T. Ray, H. Singh, A. Isaacs, and W. Smith, "Infeasibility driven evolutionary algorithm for constrained optimization," in Constraint-Handling in Evolutionary Computation, E. Mezura-Montes, Ed. Berlin: Springer, 2009, pp. $145-165$.

[21] K. Deb and R. Datta, "A bi-objective constrained optimization algorithm using a hybrid evolutionary and penalty function approach," Engineering Optimization, no. ahead-of-print, pp. 1-25, 2012.

[22] H. Myung and J. Kim, "Hybrid interior-lagrangian penalty based evolutionary optimization," in Evolutionary Programming VII. Springer, 1998, pp. 85-94.

[23] K. Deb and T. Goel, "A hybrid multi-objective evolutionary approach to engineering shape design," in Proceedings of the First International Conference on Evolutionary Multi-Criterion Optimization (EMO-01), 2001, pp. 385-399.

[24] H. Bernardino, H. Barbosa, and A. Lemonge, "A hybrid genetic algorithm for constrained optimization problems in mechanical engineering," in Evolutionary Computation, 2007. CEC 2007. IEEE Congress on IEEE, 2007, pp. 646-653.

[25] Y. Wang, Z. Cai, Y. Zhou, and Z. Fan, "Constrained optimization based on hybrid evolutionary algorithm and adaptive constraint-handling technique," Structural and Multidisciplinary Optimization, vol. 37, no. 4, pp. 395-413, 2009.

[26] R. Datta and K. Deb, "A bi-objective based hybrid evolutionary-classical algorithm for handling equality constraints," in Evolutionary MultiCriterion Optimization. Springer, 2011, pp. 313-327.

[27] K. Deb and R. Datta, "A fast and accurate solution of constrained optimization problems using a hybrid bi-objective and penalty function approach," in Proceedings of the Congress on Evolutionary Computation (CEC-2010), 2010, pp. 1-8.

[28] R. Hooke and T. A. Jeeves, "Direct search solution of numerical and statistical problems," Journal of the ACM (JACM), vol. 8, no. 2, pp. 212-229, 1961

[29] K. Deb, S. Agrawal, A. Pratap, and T. Meyarivan, "A fast and elitist multi-objective genetic algorithm: NSGA-II," IEEE Transactions on Evolutionary Computation, vol. 6, no. 2, pp. 182-197, 2002.

[30] S. Koziel and Z. Michalewicz, "Evolutionary algorithms, homomorphous mappings, and constrained parameter optimization," Evolutionary computation, vol. 7, no. 1, pp. 19-44, 1999.

[31] T. Runarsson and X. Yao, "Stochastic ranking for constrained evolutionary optimization," Evolutionary Computation, IEEE Transactions on, vol. 4, no. 3, pp. 284-294, 2000.

[32] S. Ben Hamida and M. Schoenauer, "Aschea: New results using adaptive segregational constraint handling," in Evolutionary Computation, 2002. CEC'02. Proceedings of the 2002 Congress on, vol. 1. IEEE, 2002, pp. 884-889.

[33] E. Mezura-Montes and C. Coello, "A simple multimembered evolution strategy to solve constrained optimization problems," Evolutionary Computation, IEEE Transactions on, vol. 9, no. 1, pp. 1-17, 2005.

[34] A.-R. Hedar and M. Fukushima, "Derivative-free filter simulated annealing method for constrained continuous global optimization," Journal of Global Optimization, vol. 35, no. 4, pp. 521-549, 2006.

[35] Y. Wang, Z. Cai, Y. Zhou, and W. Zeng, "An adaptive tradeoff model for constrained evolutionary optimization," IEEE Transactions on Evolutionary Computation, vol. 12, no. 1, pp. 80-92, 2008.

[36] E. Zahara and C.-H. Hu, "Solving constrained optimization problems with hybrid particle swarm optimization," Engineering Optimization, vol. 40, no. 11, pp. 1031-1049, 2008.

[37] R. Datta and K. Deb, "An adaptive normalization based constrained handling methodology with hybrid bi-objective and penalty function approach," in Evolutionary Computation (CEC), 2012 IEEE Congress on. IEEE, 2012, pp. 1-8. 